\title{
Association Analysis Identifies Risk of Ischemic Heart Disease When Temperature Increases
}

\author{
Hiroshi Morimoto ${ }^{1}$ \\ ${ }^{1}$ Hiroshi Morimoto, Graduate school of environmental study, Nagoya University, Nagoya 464, Japan. \\ Correspondence: Hiroshi Morimoto, Graduate school of environmental study, Nagoya University, Nagoya 464, Japan.
}

Received: May 9, 2016

Accepted: May 23, 2016

Available online: May 31, 2016

doi:10.11114/ijsss.v4i7.1650

URL: http://dx.doi.org/10.11114/ijsss.v4i7.1650

\begin{abstract}
The increase of mortality from ischemic heart disease has been considered to accompany falling temperature. The effects of temperature rise have been mostly neglected. The aim of this paper is to provide new insights into risk of ischemic heart disease when temperature increases. Many researchers used Poisson regression model to assess this sort of problems. Then, the cases of temperature rise were ignored as a random term in regression equations. In this article, we adopted association analysis, which highlighted the patterns of occurrence of ischemic heart disease when temperature increased. Association rule mining was performed using both daily data of emergency medical transport usage at Nagoya City in Japan and the data of weather information. The use of association analysis identified typical patterns among risky incidence of ischemic heart disease even when the weather became warmer in both winter and summer. The association rule method discovered also the importance of the weather pattern of the preceding day. These findings will suggest the existence of a mechanism, underlying the association of ischemic heart disease with a temperature rise and will contribute to better understanding for incidence of ischemic heart disease during both winter and summer.
\end{abstract}

Keywords: ischemic heart disease, myocardial infarction, weather, association analysis

\section{Introduction}

Health risks associated with exposure to cold were well documented. The cold-related increases of mortality of ischemic heart disease have been reported in many studies. For example, mortality of myocardial infarctions was announced to have increased to a greater extent with given fall of temperature (The Eurowinter Group, 1997). They estimated precisely the percentage increases in deaths per day per $1{ }^{\circ} \mathrm{C}$ fall in temperature below $18^{\circ} \mathrm{C}$ (indices of cold-related mortality) by generalized linear modeling.

In examining the problem of cold as a risk factor, the evidence of coronary blood flow and the induction thrombogenesis was discussed in literature. The influence of cold exposure to coronary blood flow was observed by (Muller, Gao, McQuillan, Leuenberger, \& Sinoway, 1997). They showed that cold air breathing significantly influenced efferent control of coronary blood flow. Myocardial infarctions are induced by the thrombogenesis in coronary vein. In this point, short-term cold exposure in young healthy subjects caused changes in hematological factors known to be associated with the promotion of thrombogenesis (Mercer, Østerud, \& Tveita, 1999). The greatest number of cases have been found to occur during the winter months. Therefore it has been believed that acute myocardial infarction is more common in winter and more common on colder days.

On the other hand, there is a shortage of published research describing the relationships between the risk of acute myocardial infarctions and environmental exposure to heat. There was an old report showing that the greatest number of cases of myocardial infarctions occurred in the summer months, and the lowest number of cases in the winter months in Texas (Heyer,Teng, \& Barris, 1953). There was also a study that addressed an increased risk of AMI admissions to hospital during hot weather in Australia (Loughnan, Nicholls, \& Tapper, 2010). But they focused on the discussion on the relationship of socioeconomic circumstance and acute myocardial infarction. As for cerebral infarction, a new aspect of this problem was recently provided by Morimoto (2015b). It showed that there existed significant patterns for the incidence of cerebral infarction when temperature increased.

The increase of temperature may cause the following process. The rise of temperature may militate for widening of a blood vessel (Kellogg, Zhao, \& Wu, 2009), and make blood pressure to drop. The decrease of blood pressure has a 
tendecy to lead to myocardial infarction (Luukinen, Koski, Laippala, \& Airaksinen, 2004). The further details of mechanism were explored in discussion section. This suggests the existence of a link between the increase of temperature and the incidence of ischemic heart disease.

In the present paper, we applied association analysis to discover the relationship between rise of temperature and incidence of ischemic heart disease in both summer and winter. More generally, we tried to find association rules that might connect the change of the weather state (including the rise of temperature) to the increase of patients of ischemic heart disease.

\section{Methods}

In the present paper, we extracted three types of data: the data of emergency transport, the data of weather elements (such as mean temperature) and the data of weather patterns. The data of emergency transport were supplied by Nagoya City Fire Department. These were daily data from 2002 January up to 2005 December in Nagoya City in Japan. It contained the number of patients who were first transported by ambulance to hospitals and then diagnosed ischemic heart disease (including myocardial infarction and angina pectoris) at the hospitals.

The weather data were a daily data of various whether elements, such as temperature (mean, maximum and minimum temperature) and the hours of sunshine, which were supplied by Japan Meteorological Agency. The data of weather patterns were extracted from Japan Weather Association (JWA), which were called "11-types of weather patterns" including the pattern of "high pressure", "a cold front" and so on. See for details Morimoto (2015b).

Association rule discovery is a general-purpose rule-discovery scheme and has broad applications and is a well-researched technique of data mining. It has been widely accepted because of the simplicity of the problem statement and the effectiveness of pruning by specific index, such as "lift". It aims to extract frequent patterns in data set.

Association rules were originally developed with the purpose of market-basket analysis, where it was of interest to find patterns of the form X -> Y, e.g., beer -> peanuts, with the intuitive meaning "baskets that contain X tend to contain Y". See for details Ordonez (2006) and Li (2006).

Here in the present paper, we will avoid the notations of "basket" and will use more mathematical notions (cf. Morimoto, 2015 b). We therefore begin with a database $D$ of a form like a matrix:

$$
D=\left\{d_{i j}\right\}
$$

For example, in later sections, we consider $D$ such that the row labels are dates (from 2002/1/1 to 2005/12/31) and the column labels are the information of the number of patients of ischemic heart disease, weather elements and so on. Here, "patients" means those people who were transported by emergency and were diagnosed with ischemic heart disease.

Let $T$ be a set of all the row vectors of $D$ :

$$
T=\left\{d_{1}, d_{2, \ldots}\right\}, d_{\mathrm{i}}=\left(d_{\mathrm{i} 1}, \quad d_{\mathrm{i} 2}, \ldots, d_{\mathrm{ij}}, \ldots\right)
$$

Then the set $T$ might be considered as a set of "transactions" in the context of basket analysis. The "items" are like mean temperature, maximum temperature, weather pattern, number of patients and so on.

We now pay attention to column (say j-th column) of each row vector Let us fix column number $j$ and fix some possible value of $j$-th column, say $x$. With given $j$ and $x$, let $X$ be as the subset of $T$ satisfying the following:

$$
X=\left\{d_{\mathrm{i}} \text { in } T \mid \text { the value of the column } \mathrm{j} \text { of } d_{\mathrm{i}} \text {, i.e., } d_{\mathrm{ij}} \text { is equal to the value } x\right\} \text {. }
$$

The condition in the above definition is called a "rule" corresponding to $X$. We also denote this rule as $X$, as far as there is no misunderstanding,

$$
X=\left\{\text { the value of the column } \mathrm{j} \text { of } d_{\mathrm{i}} \text {, i.e., } d_{\mathrm{ij}} \text { is equal to the value } \mathrm{x}\right\} .
$$

Therefore $X$ is considered as a subset of $T$, and considered also as a rule at the same time.

For example, in our case, $X$ can be a condition like

$\{$ the mean temperature of the day in question is equal to 10$\}$.

Let $Y$ be another condition, like

$\left\{\right.$ the value of the column $k$ of $d_{\mathrm{i}}$, i.e., $d_{\mathrm{ik}}$ is equal to $y$ \}.

An expression $\{X=>Y\}$ is called an association rule. That means

"If $X$ is satisfied then $\mathrm{Y}$ is satisfied".

The condition $X$ is called "antecedent" and $Y$ is called "consequent". 
For given $X$, the probability $\mathrm{P}(X)$ is defined as a fraction of the number of rows of $T$ that satisfies the rule $X$ over the total number of $T$. This is equal to the probability that the rule $X$ is satisfied.

$$
\mathrm{P}(X)=\#(\text { row of } T \text { that satisfies } X) / \#(T)
$$

where \#( set ) denotes the number of the set.

There are three basic indexes, in association analysis, that estimates whether the rules found are acceptable or unrealistic. These three measures are called "support", "confidence" and "lift".

Now let $\{\mathrm{X}=>\mathrm{Y}\}$ be an association rule. The "confidence" of rule $\{X \Rightarrow Y)$, denoted by $\operatorname{conf}(X=>Y)$, is defined as the

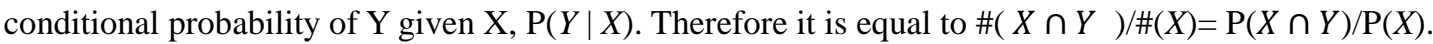

The "lift" of the rule $\{X \Rightarrow Y\}$ is defined by

$$
\operatorname{lift}(X \Rightarrow>Y)=\operatorname{conf}(X \Rightarrow Y) / \mathrm{P}(Y)=[\mathrm{P}(X \cap Y)] /[\mathrm{P}(X) \mathrm{P}(Y)]
$$

If the lift is equal to 1 , then $\mathrm{P}(X \cap Y)=\mathrm{P}(X) \mathrm{P}(Y)$, i.e., the condition $X$ is independent from Y. This implies the rule $\{X \Rightarrow$ $Y\}$ is not effective. If the lift is greater than 1 , then the condition $X$ is meaningful and the rule $\{X \Rightarrow Y\}$ can be estimated to be a effective rule. Therefore, "confidence" and "lift" are effectual measures to quantify association rules.

The "support" of the rule $\{\mathrm{X} \Rightarrow \mathrm{Y}\}$ is defined as the probability of $X \cap Y$, and denoted by $\sup (\mathrm{X}=>\mathrm{Y})$, which is then

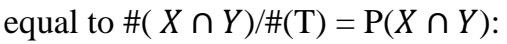

$$
\sup (\mathrm{X}=>\mathrm{Y})=\mathrm{P}(X \cap Y) .
$$

This definition of support is in accordance with the way of the software $\mathrm{R}$ that was used in this paper.

The antecedent condition $X$ be a combination of several conditions, such as " $X_{1}$ and $X_{2}$ " or denoted by

$$
\left\{X_{1}, X_{2}\right\}
$$

where "," means "and".

In the present paper, $T$ is supposed to be a set of row vectors labeled with dates from 2002/1/1 to 2004/12/31. The items of $T$ are composed of weather elements (e.g., minimum temperature), weather patterns and the risk of ischemic heart disease (i.e., the number of patients who were diagnosed as ischemic heart disease after transport to hospital). Then for example, one possible rule could be an association rule like

\{The temperature is very low, the weather pattern of the previous day is a type of low pressure\}

$$
\Rightarrow \text { the risk of ischemic heart disease is high }\} \text {. }
$$

If the "confidence" and "lift" are relatively large then the association rule can be regarded as a good rule. Heuristic rule discovery is usually expected to produce a small set of accurate rules. It generates all rules satisfying some constraints, but yields too many rules and the most of them are infeasible. To settle this problem, the notions such as "lift" or "support" are useful to distinguish practical rules from ineffective rules.

\section{Results}

The parameter "temperature" has been likely to be selected as one of weather elements for various analysis of disease, because the selection of other elements caused a subtle problem. In Morimoto 2015b, the "sea-level pressure" was selected for the analysis of cerebral infarction, adding to temperature. Here in the present paper, for the analysis of ischemic heart disease, we selected "the hours of sunshine", as another weather element, besides temperature.

The parameter, "the hours of sunshine", was chosen here, because it marked good value for the analysis of variance. We divided our daily data into three classes, "low risk", "medium risk", "high risk", due to the number of patients admitted as ischemic heart disease. Then we applied the analysis of variance to these three classes according to the daily difference of hours of sunshine. This test of variance was useful to bring correlation among risk of ischemic heart disease and sunshine. The result of p-values of variance test was shown in Table 1. From June to December, the daily difference of hours of sunshine could be regarded as a good indicator for the risk of ischemic heart disease.

Table 1. Analysis of variance for the difference of hours of sunshine

\begin{tabular}{lcccccccccccc}
\hline month & 1 & 2 & 3 & 4 & 5 & 6 & 7 & 8 & 9 & 10 & 11 & 12 \\
\hline p-value for sunshine & 0.99 & 0.38 & 0.25 & 0.5 & 0.26 & 0.17 & 0.19 & 0.25 & 0.19 & 0.1 & 0.06 & 0.16 \\
\hline
\end{tabular}

Description: The p-values were calculated for three months including the month indicated. For example, "month 11" means the collection of October, November and December. This was due to the shortage of data for only one month.

Before applying the method of association analysis, we must process the original data including the data of weather elements, patients of ischemic disease and classified weather patterns. We made a new database from these data to fit 
the association rules mining. We constructed a new database from all the data in summer (June, July and August) from 2002 to 2005, and then later from all the data in winter (November, December and January) later.

We first calculated the differences of weather elements between two consequent days. For example, we computed the mean temperature of 2002/6/2 minus the mean temperature of 2002/6/1. We named this value as mean temperature difference on 2002/6/2 (abbreviated as "meantmp_d" in Table 2). In Table 2, the values of "maxtmp_d, mintmp_d, sunshine_d" were obtained in a similar way from the differences of the values of maximum temperature , minimum temperature and hours of sunshine. As weather elements, we chose temperature (such as mean, maximum and minimum of temperature) and the hours of sunshine.

The data of weather patterns, called "11-types of weather patterns", were those given by Japan Weather Association (JWA). The data included the pattern of "high pressure", "a cold front" and so on. These 11-types were expressed as numbers from 1 to 11. See for details (Morimoto, 2015b). The weather pattern of previous day was added to Table 2, together with the pattern of the day in question (current day). These were denoted by "previouspat" and "pattern". We also added to Table 2 the number of patients who were diagnosed as ischemic heart disease after transport to hospital. As a result of this process, our new data began on the date 2002/6/2 (for summer data), and the first part of our new data was listed in Table 2. For winter data, the data begins on 2002/12/2.

Table 2. The first default data of our study.

\begin{tabular}{cccccccc}
\hline date & meantmp_d & maxtmp_d & mintmp_d & sunshine_d & previouspat & pattern & ischemic \\
\hline $2002 / 6 / 2$ & 1.6 & 1.5 & 2.2 & 1.6 & 6 & 6 & 15 \\
$2002 / 6 / 3$ & -1 & 0.4 & -1.8 & -3 & 6 & 6 & 8 \\
$2002 / 6 / 4$ & 0.9 & 0.2 & 0.9 & -0.2 & 6 & 6 & 10 \\
$2002 / 6 / 5$ & 1.4 & 2.6 & 1.7 & 2.4 & 6 & 1 & 5 \\
$2002 / 6 / 6$ & -1.4 & -4.1 & 1.7 & -5.2 & 6 & 6 & 13 \\
$2002 / 6 / 7$ & 0.2 & 2 & -2.2 & 2.2 & 6 & 6 & 8 \\
\hline
\end{tabular}

Description: In the above table, "meantmp_d" means the difference of mean temperature between two days. "pattern" means the weather pattern (or chart). "previouspat" means the weather pattern of previous day (one day earlier day).

In order to make association analysis to be effective, we categorized these numbers in Table 2 into several categories. For weather elements, we categorized into three categories "up", "down" or "even": "up" for numbers of plus sign, "down" for numbers of minus sign and "even" for nearly equal numbers. Threshold for "evenness" was set to about $10 \%$ of maximum absolute values.

For weather charts, we classified 11-types of weather patterns into two simple patterns, "high pressure type" and "low pressure type". We abbreviated these as "highp" and "lowp" in Table 3. In fact, we replaced the types 3,4,5,6 to high pressure type and 1,2,7,8,9,10,11 to low pressure type. For the number of patients, we simplified them as "high risk", "low risk" and "mid risk". The thresholds of this classification were determined by calculating distribution of the patients numbers, and were defined so that the ratio of low risk (and high risk too) occupied 25\% and mid risk 50\%. Thus if the number of patients was less than or equal to 2, then the risk was set the risk "low". Finally the data were simplified as listed in Table 3. The data had 8 columns and about 360 rows (daily data in summer during four years from 2002 to 2005).

Table 3. The processed data which were used for association analysis.

\begin{tabular}{cccccccc}
\hline date & meantmp_d & maxtmp_d & mintmp_d & sunshine_d & previouspat & pattern & risk \\
\hline $2002 / 6 / 2$ & up & up & up & even & highp & highp & high \\
$2002 / 6 / 3$ & down & even & down & down & highp & highp & mid \\
$2002 / 6 / 4$ & even & even & even & even & highp & highp & mid \\
$2002 / 6 / 5$ & up & up & up & up & highp & lowp & low \\
$2002 / 6 / 6$ & down & down & up & down & lowp & highp & high \\
$2002 / 6 / 7$ & even & up & down & up & highp & highp & mid \\
\hline
\end{tabular}

Description: Here "lowp" (or "highp") expressed that the weather chart was of low (or high) pressure type.

The software used in this paper for association rules mining was a software " $R$ " with the package "arules". We executed our association analysis for both summer and winter data from 2002 to 2005. Since the whole rules found were 
extremely large, we only listed, in Table 4, six rules with high score in "lift" for the summer data. Similarly, for winter data, ten rules with high score in "lift" were listed in Table 5.

Table 4. The result of association rule mining for summer form 2002 to 2005

\begin{tabular}{|c|c|c|c|c|c|c|c|}
\hline & lhs & & rhs & support & confidence & lhs.support & lift \\
\hline 1 & $\begin{array}{l}\{\text { sunshine_d=up, } \\
\text { previouspat=highp }\}\end{array}$ & $\Rightarrow$ & $\{$ risk=high $\}$ & 0.04632153 & 0.4358974 & 0.10626703 & 1.683941 \\
\hline 2 & $\begin{array}{l}\{\text { meantmp_d=up, } \\
\text { previouspat=highp }\}\end{array}$ & $\Rightarrow$ & $\{$ risk=high $\}$ & 0.03542234 & 0.4333333 & 0.08174387 & 1.674035 \\
\hline 3 & $\begin{array}{l}\{\text { maxtmp_d=up, } \\
\text { previouspat=highp }\}\end{array}$ & $\Rightarrow$ & $\{$ risk=high $\}$ & 0.03269755 & 0.4137931 & 0.07901907 & 1.598548 \\
\hline 4 & $\begin{array}{l}\{\text { maxtmp_d=up, } \\
\text { mintmp_d=up }\}\end{array}$ & $\Rightarrow$ & $\{$ risk=high $\}$ & 0.02997275 & 0.4074074 & 0.07356948 & 1.573879 \\
\hline 5 & $\begin{array}{l}\{\text { maxtmp_d=even, } \\
\text { sunshine_d=up }\end{array}$ & $\Rightarrow$ & $\{$ risk $=$ high $\}$ & 0.04632153 & 0.3777778 & 0.1226158 & 1.459415 \\
\hline 6 & $\begin{array}{l}\{\text { meantmp_d=up, } \\
\text { mintmp_d=up }\}\end{array}$ & $\Rightarrow$ & $\{$ risk $=$ high $\}$ & 0.03269755 & 0.375 & 0.08719346 & 1.448684 \\
\hline
\end{tabular}

Description: The rules found were arranged according to the value of "lift".

In Table 4, most of the conditions of left hand sides of the rules ("antecedent") contained the rise of some temperature (mean, minimum or maximum) or the increase of the hours of sunshine (the rule no.5). Among six rules, the top three rules contained that the weather charts of the previous day was of high pressure type.

In Table 5, similar rules were observed. Among ten rules, most of left hand sides of the rules (i.e., antecedent) contained the increase of certain temperature and the hour of sunshine, except the number 2, 3, and 5. In number 7, the condition of left hand side contained the high pressure type of previous day. This is remarkable because in general the cold exposure was believed to be a trigger for ischemic heart disease. The high risk of rise of temperature in winter was observed also for cerebral infarctions in (Morimoto, 2015b).

Thus we noticed that the rise of temperature led to high risk of ischemic heart disease including myocardial infarctions. The weather charts of the previous day also had an effect on the risk for the incidence of ischemic heart disease. In particular, a type of high pressure might become a trigger to ischemic heart disease.

Table 5. The result of association rule mining for winter form 2002 to 2005

\begin{tabular}{|c|c|c|c|c|c|c|c|}
\hline & lhs & & rhs & support & confidence & lhs.support & lift \\
\hline 1 & $\begin{array}{l}\{\text { mintmp_d=up, } \\
\text { pattern=highp }\}\end{array}$ & $\Rightarrow$ & $\{$ risk $=$ high $\}$ & 0.06944444 & 0.3846154 & 0.18055556 & 1.331361 \\
\hline 2 & $\begin{array}{l}\{\text { mintmp_d=down, } \\
\text { sunshine_d=down }\}\end{array}$ & $\Rightarrow$ & $\{$ risk $=$ high $\}$ & 0.02777778 & 0.3846154 & 0.07222222 & 1.331361 \\
\hline 3 & $\begin{array}{l}\{\text { maxtmp_d=even, } \\
\text { sunshine_d=down }\end{array}$ & $\Rightarrow$ & $\{$ risk $=$ high $\}$ & 0.06388889 & 0.3833333 & 0.16666667 & 1.326923 \\
\hline 4 & $\begin{array}{l}\{\text { maxtmp_d=even, } \\
\text { mintmp_d=up\} }\end{array}$ & $\Rightarrow$ & $\{$ risk $=$ high $\}$ & 0.04444444 & 0.3809524 & 0.11666667 & 1.318681 \\
\hline 5 & $\begin{array}{l}\{\text { maxtmp_d=even, } \\
\text { mintmp_d=down }\}\end{array}$ & $\Rightarrow$ & $\{$ risk $=$ high $\}$ & 0.055555566 & 0.3703704 & 0.15 & 1.282051 \\
\hline
\end{tabular}




\begin{tabular}{|c|c|c|c|c|c|c|c|}
\hline 6 & $\begin{array}{l}\{\text { meantmp_d=up, } \\
\text { maxtmp_d=even }\}\end{array}$ & $\Rightarrow$ & $\{$ risk=high $\}$ & 0.03055556 & 0.3666667 & 0.08333333 & 1.269231 \\
\hline 7 & $\begin{array}{l}\{\text { mintmp_d }=\text { up }, \\
\text { previouspat=highp }\}\end{array}$ & $\Rightarrow$ & $\{$ risk=high $\}$ & 0.08333333 & 0.3658537 & 0.22777778 & 1.266417 \\
\hline 8 & $\begin{array}{l}\{\text { maxtmp_d=up, } \\
\text { mintmp_d=up }\}\end{array}$ & $\Rightarrow$ & $\{$ risk=high $\}$ & 0.03333333 & 0.3636364 & 0.09166667 & 1.258741 \\
\hline 9 & $\begin{array}{l}\{\text { mintmp_d=up, } \\
\text { sunshine_d=up }\}\end{array}$ & $\Rightarrow$ & $\{$ risk=high $\}$ & 0.02222222 & 0.3636364 & 0.06111111 & 1.258741 \\
\hline 10 & $\begin{array}{l}\{\text { maxtmp_d=even, } \\
\text { sunshine_d=up }\}\end{array}$ & $\Rightarrow$ & $\{$ risk=high $\}$ & 0.04722222 & 0.3617021 & 0.13055556 & 1.252046 \\
\hline
\end{tabular}

We noticed some common features of high risk for ischemic heart disease in the list of Table 4 and 5. The risky days were identified when temperature and the hours of sunlight increased. For weather patterns or charts, the state of previous day had some effect on the risk. These findings suggested that there existed characteristic pattern among risky days in both winter and summer.

\section{Discussion}

We applied association analysis to the data consisting of the numbers of ischemic patients, weather conditions and weather charts (or patterns). This association analysis highlighted the following typical weather conditions that triggered the onset of ischemic heart disease (see Table 4 and 5). Most of the conditions shared the following features:

(1) A rise in temperature (mean, maximum or minimum),

(2) An increase in hour of sunshine, and

(3) A high atmospheric pressure weather pattern on the previous day.

Mechanisms linking the rise in temperature to the onset of ischemic heart disease have remained speculative. Here we offer two possible explanations of the assumed mechanisms. The first was connected to hypotension and the second was to dehydration.

It was known that the exposure to heat led to widening of a blood vessel or vasodilatation (Kellogg, Zhao, \& Wu, 2009). Then this vascular enlargement induced vasodilatation and hence hypotension. This hypotension had tendency to cause promotion of clots or thrombosis (Rezende-Neto 2010; Berroir, Grabli, Héran, Bakouche, \& Bousser, 2004; Wouter \& Menahem, 2008). The drop of blood pressure was reported to lead to myocardial infarction (Luukinen, Koski, Laippala, \& Airaksinen, 2004).

The other possible way of effects of rise of temperature to ischemic heart disease is the aspect of dehydration. It is well-known that the rise of temperature induces dehydration. It was reported that some blood flow was reduced with dehydration (Gonzalez-Alonso, Calbet, \& Nielsen, 1998). The dehydration had a tendency to make the blood sticky and mushy. As a result, the vein might become thrombosed and suffer a blood colt. Therefore the coronary artery had to suffer to be blocked by thrombosis.

As for the weather pattern, we listed a typical weather pattern of the previous day in the condition (3) which was found to be common among association rules.

The frequencies of myocardial infarction and of sudden cardiac death were highest early in the morning (Tofler et al., 1987). In particular, the incidence of ischemic heart disease had a tendency to occur when we started our daily works in the early morning. This could be the reason why the weather pattern, such as high pressure type of the previous day, appeared in association rules.

\section{Conclusion}

Cold exposure had been considered as a trigger of mortality attributed to ischemic heart disease, such as myocardial infarction and angina pectoris. We applied the theory of association rule mining to the daily data of emergency ambulance transport in Nagoya City in Japan. Association rule mining revealed the existence of rules that linked a rise 
in temperature to an increase of the risk of ischemic heart disease.

This association analysis highlighted the existence of the following rules:

If (1) The temperature (mean, maximum or minimum) rise,

(2) The hour of sunshine increased, and

(3) The weather pattern of the previous day was covered with high atmospheric pressure,

then, the risk of ischemic heart disease increases.

Causal mechanisms linking a rise of temperature to the onset of ischemic heart disease may be attributed to both hypotension and dehydration caused by a rise in temperature.

\section{Acknowledgements}

We thank Nagoya City Fire Department and Japan Weather Association (JWA) for the offer of the daily data of ambulance transport in Nagoya City and 11-types of weather patterns respectively.

\section{References}

Berroir, S., Grabli, D., Héran F., Bakouche P., \& Bousser, M. G. (2004). Cerebral Sinus Venous Thrombosis in Two Patients with Spontaneous Intracranial Hypotension. Cerebrovascular diseases, 17(1), 9-12. http://dx.doi.org/10.1159/000073892

Gonzalez-Alonso, J., Calbet, J. A. L., \& Nielsen, B. (1998). Muscle blood flow is reduced with dehydration during prolonged exercise in humans. The Jounal of Physiology, 513(3), 895-905. http://dx.doi.org/10.1111/j.1469-7793.1998.895ba.x

Heyer, H. E.,Teng, H. C., \& Barris, W. (1953). The increased frequency of acute myocardial infarction during summer months in a warm climate: A study of 1,386 cases from Dallas, Texas. American Heart Journal, 45(5), 741-748. http://dx.doi.org/10.1016/0002-8703(53)90315-9

Kellogg, Jr. D. L., Zhao, J. L., \& Wu, Y. (2009). Roles of nitric oxide synthase isoforms in cutaneous vasodilation induced by local warming of the skin and whole body heat stress in humans. Journal of Applied Physiology, 107(5), 1438-1444. http://dx.doi.org/10.1152/japplphysiol.00690.2009

Li, J. (2006). On optimal rule discovery. IEEE Transactions on Knowledge and Data Engineering, 18(4), http://dx.doi.org/10.1109/TKDE.2006.1599385

Loughnan, M., Nicholls, N., \& Tapper, N. J. (2010). The effects of summer temperature, age andsocioeconomic circumstance on Acute Myocardial Infarction admissions in Melbourne, Australia. International Journal of Health Geographics, 9(41). http://dx.doi.org/10.1186/1476-072X-9-41

Luukinen, H., Koski, K., Laippala, P., \& Airaksinen, K. E. J. (2004). Orthostatic hypotension and the risk of myocardial infarction in the home-dwelling elderly. Journal of Internal Medicine, 255(4), 486-493. http://dx.doi.org/10.1111/j.1365-2796.2004.01313.x

Marchant, B., Ranjadayalan, K., Stevenson, R., Wilkinson, P., \& Timmis, A. D. (1993). Circadian and seasonal factors in the pathogenesis of acute myocardial infarction: the influence of environmental temperature. British Heart Journal, 69, 385-387. http://dx.doi.org/10.1136/hrt.69.5.385

Mercer, J. B., Østerud, B., \& Tveita, T. (1999). The Effect of Short-Term Cold Exposure on Risk Factors for Cardiovascular Disease. Thrombosis Research, 95(2), 93-104. http://dx.doi.org/10.1016/S0049-3848(99)00028-6

Morimoto, H. (2015a). Study on Links between Cerebral Infarction and Climate Change Based on Hidden Markov Models. International Journal of Social Science Studies, 3(5), 180-186. http://dx.doi.org/10.11114/ijsss.v3i5.

Morimoto, H. (2015b). Patterns in Stroke Occurrence on Warm Days in Winter by Associations Analysis. Open Journal of Applied Sciences, 5, 776-782. http://dx.doi.org/10.4236/ojapps.2015.512074

Morimoto, H. (2016) .Hidden Markov Models and Self-Organizing Maps Applied to Stroke Incidence Open Journal of Applied Sciences, 6, 158-168. http://dx.doi.org/10.4236/ojapps.2016.63017

Muller, M. D., Gao, Z., McQuillan, P. M., Leuenberger, U. A., \& Sinoway, L. I. (2014). Coronary responses to cold air inhalation following afferent and efferent blockade. American Journal of Physiology - Heart and Circulatory Physiology, 307(2), H228-H235. http://dx.doi.org/10.1152/ajpheart.00174.2014

Ordonez, C. (2006). Association rule discovery with the train and test approach for heart disease prediction. Information Technology in Biomedicine, 10(2), 334-343. http://dx.doi.org/10.1109/TITB.2006.864475 
Rezende-Neto, J. B., Rizoli, S. B., Andrade, M. V., Ribeiro, D. D., Lisboa, T. A., Camargos, E. R., Martins, P., \& Cunha-Melo, J. R. (2010) . Permissive Hypotension and Desmopressin Enhance Clot Formation. Journal of Trauma-Injury Infection \& Critical Care, 68(1), 42-51. http://dx.doi.org/10.1097/TA.0b013e3181c66393

The Eurowinter Group (Correspondence to Keatinge, K. R.) (1997). Cold exposure and winter mortality from ischaemic heart disease, cerebrovascular disease, respiratory disease, and all causes in warm and cold regions of Europe. The Lancet, 349(9062), 1341-1346. http://dx.doi.org/10.1016/S0140-6736(96)12338-2

Tofler, G. H., Brezinski, D., Schafer, A. I., Czeisler, C. A., Rutherford, J. D., Willich, S. N., Gleason, R. E., Williams, G. H., \& Muller, J. E. (1987). Concurrent Morning Increase in Platelet Aggregability and the Risk of Myocardial Infarction and Sudden Cardiac Death. New England Journal of Medicine, 316, 1514-1518. http://dx.doi.org/10.1056/NEJM198706113162405

Wouter I. S., \& Menahem M. M. (2008). Cerebral Venous Thrombosis in Spontaneous Intracranial Hypotension. Headache: The Journal of Head and Face Pain, 48(10), 1511-1519.

http://dx.doi.org/10.1111/j.1526-4610.2008.01251.x

\section{(cc) $\mathrm{BY}$}

This work is licensed under a Creative Commons Attribution 3.0 License. 